\section{COMMON ARTIFACTS OF PULMONARY ARTERY AND PULMONARY ARTERY WEDGE PRESSURES: RECOGNITION AND INTERPRETATION}

Edward A. Schmitt, MD, and C. O. Brantigan, MD, FACS, FCCP
From the Department of Surgery, Presbyterian/St. Luke's Medical Center, and St. Joseph Hospital, Denver, CO.

Received Dec 24, 1984, and in revised form Aug 14, 1985. Accepted for publication Sep 5, 1985.

Address correspondence to Dr Brantigan, 1746 Lafayette St, Denver, $\mathrm{CO}, 80218$.
Schmitt EA, Brantigan CO. Common artifacts of pulmonary artery and pulmonary artery wedge pressure: Recognition and interpretation.

J Clin Monit 1986;2:44-52

ABSTRACT. Bedside measurement of pulmonary artery pressure and pulmonary artery wedge pressure has an important role in the management of critically ill patients. Unfortunately, waveform abnormalities and artifacts commonly distort numeric values and lead to incorrect therapeutic decisions. The clinical impact of these artifacts is magnified by the digital pressure displays used in most intensive care units. We present here an atlas and an analysis of the artifacts that commonly occur. Use of analog rather than digital pulmonary artery wedge pressure data, when combined with an understanding of the physiological characteristics of patients, can prevent critical errors in patient management.

KEY WonDS. Lung: pulmonary artery pressure; pulmonary artery wedge pressure. Monitoring: blood pressure, artifact

Since the introduction of Swan-Ganz cardiac catheters [1], cardiac catheterization has become a frequently performed bedside procedure in intensive care units. The resulting hemodynamic data provide a picture of the patient's status that may be invaluable. Data are displayed on highly technological digital bedside monitors. Unfortunately, digital readouts may detect simply the highs and lows during some standard interval and produce data that are entirely artifactual. Hence, measurements designed to reassure the physician or lead to accurate decisions instead frequently become counterproductive. Analysis of an analog strip-chart recording of the pulmonary artery wedge pressure tracing and observation of the status of each individual patient can eliminate artifact from these vital measurements. We present here an atlas of the common artifacts, an analysis of how they occur, and a simple way to handle them using a calibrated analog recorder.

\section{MATERIALS AND METHODS}

Patients for this study were selected from the intensive care unit at Denver Presbyterian Hospital of Presbyterian/St Luke's Medical Center when the bedside monitor indicated a high probability of important artifact in pulmonary artery or pulmonary artery wedge pressures. Digital readouts made by a model \#3474CS4JK digital readout computer (General Electric) were compared with an analog reading of pulmonary artery and pulmonary artery wedge pressures obtained with a model RI-5DC recorder (General Scanning, Inc). This device interfaced well with the General Electric system. We wished to obtain characteristic examples, therefore, no attempt was made to assess the frequency of 
significant differences between the methods; however, we did note that artifacts of varying importance occurred in the majority of the patients in the intensive care unit. All standard precautions were taken to assure that accurate data would be obtained by both methods. Proper placement of the pulmonary artery pressure catheter was ascertained, and the pressure lines to the patient were examined to make certain that they were free of air bubbles. The transducers were inspected to make certain that they were free of air and that they contained an appropriate amount of water. The analog strip recorder was carefully zeroed to atmospheric pressure at precisely the same altitude as the patient's heart. The patients were supine in bed during the recordings. The digital readout computer provided output exactly as it was designed to on all patients, and agreement with the chart recorder was excellent. However, the digital display always interpreted artifacts or abnormal pressure waves that satisfied the programmed criteria as the pulmonary artery systolic, diastolic, or wedge pressures. This occurred because the algorithm for digital display looks at a segment of the pressure tracing, identifies the highest value seen during that interval as systolic, the lowest as diastolic, and continuously averages the waveform to produce a mean. The "mean" reading is usually used to indicate the pulmonary artery wedge pressure. While other, more advanced, algorithms improve on this simplistic approach [2], our monitors demonstrate the problem intrinsic to all such digital displays; namely, that they are unable to completely eliminate artifactual waveforms from consideration in their associated algorithms. We recommend interpretation of the calibrated analog wave and reading the pulmonary artery wedge pressures at end expiration.

The "wedge" state is defined in this paper as the state in which the inflated catheter balloon appears to just occlude the pulmonary artery. We attempted to identify each pressure wave seen on a pulmonary artery wedge pressure tracing and selected as the "true wedge" the portion of the tracing that most closely approximated left ventricular filling pressure. Occasionally, tracings are uninterpretable and must be recognized as such. Uninterpretable tracings most commonly occur when heart rate and respiratory rate are similar. More often there is a relatively stable portion of the trace in the midst of artifacts and abnormal pressure waves that appears to approximate left ventricular filling pressure. When significant $V$ waves are present we read the wedge at the valley of these tracings; we believe that the regurgitant wave does not represent ventricular filling pressure. In the case of respiratory artifacts we attempt to read the values at end expiration as this is the point at which atmospheric and alveolar pressure values are most nearly equal. Although most respiratory artifacts are easily identified from examining the tracing or by watching the patient while data are collected, direct airway pressure recordings are helfpul. Most intensive care units do not have the capability for measuring airway pressures continuously, however, and in our experience such recordings are usually not necessary. The examples given in Figures 1-12, all recorded at a chart speed of 5 $\mathrm{mm} / \mathrm{s}$, demonstrate how digital displays lead to errors and illustrate how to interpret an analog waveform.

\section{RESULTS}

Figure 1 shows a negative respiratory artifact. The patient had labored breathing secondary to pneumonia, sepsis, and pulmonary edema. The wedge pressure tracing $(\mathrm{PAW})$ demonstrates large negative excursions $(\mathrm{A})$; hence, the mean wedge pressure (an average of high and low when determined digitally) was less than 0 . The actual wedge pressure was approximately $10 \mathrm{~mm} \mathrm{Hg}$ and could have been accurately measured by setting the digital readout to "systolic." A similar distortion was produced in the pulmonary artery diastolic (PAD) tracing. The true pulmonary artery systolic pressure (PAS) is marked by small arrows. The true diastolic pressure, identified by the large arrows, was approximately 30 $\mathrm{mm} \mathrm{Hg}$. The digital equipment interpreted the pulmonary artery diastolic pressure at $12 \mathrm{~mm} \mathrm{Hg}$. This artifact is seen commonly in patients who generate a large negative intrathoracic pressure to breathe, and can occur when patients are either on or off the ventilator.

Figure 2 shows a positive respiratory artifact. This is the pulmonary artery pressure tracing of a patient receiving controlled ventilation. The patient was making no attempt to breathe on his own, but each time the respirator cycled it created positive intrapleural pressure that produced an artifact (A). The artifact was averaged by the digital equipment into the wedge pressure tracing, producing a falsely high reading. The pulmonary artery systolic pressure was distorted in the same fashion. Whereas the digital equipment interpreted the systolic pressure as $50 \mathrm{~mm} \mathrm{Hg}$, it was actually only $40 \mathrm{~mm}$ $\mathrm{Hg}$.

Placing the patient whose tracing is shown in Figure 1 on a mechanical ventilator, such as was done in the second example, could change a falsely low pulmonary artery wedge pressure to a falsely high one. Thus the act of placing a patient on a ventilator could easily cause an increase of $20 \mathrm{~mm} \mathrm{Hg}$ in the wedge pressure displayed by digital equipment, when in fact there is no marked change in the patient's hemodynamic status. 


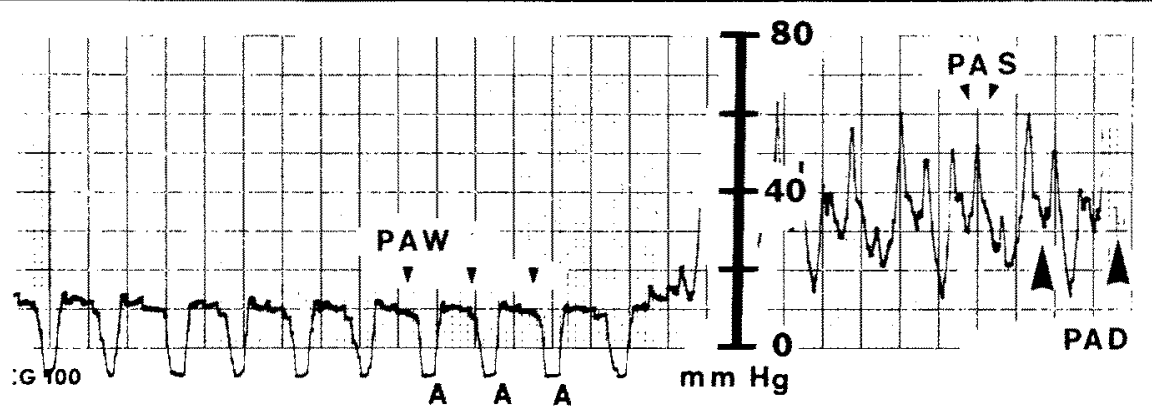

Fig 1. Negative inspiratory artifact (A), in a patient with labored breathing from pneumonia, sepsis, and pulmonary edema. The catheter balloon was deflated in the tracing on the right, and inflated in the tracing on the left. PAW = pulmonary artery wedge; $\mathrm{PAS}=$ pulmonary artery systolic; $\mathrm{PAD}=$ pulmonary artery diastolic.

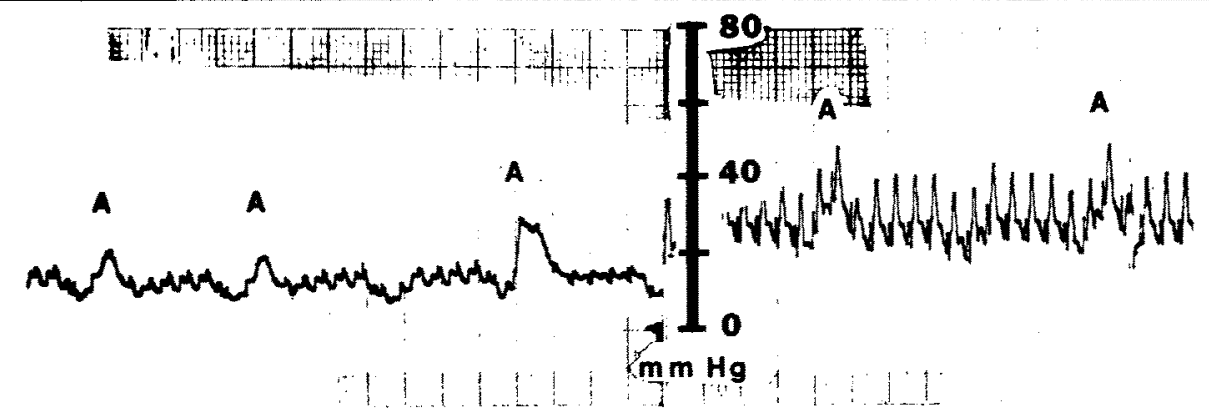

Fig 2. Positive respiratory artifact (A) due to controlled ventilation. In the left tracing the catheter balloon was inflated; in the right tracing, it was deflated.

Figure 3 shows positive and negative artifacts occurring together. The patient had stiff lungs and labored respiration and was receiving assisted ventilation. The wedge pressure tracing was distorted by a $24-\mathrm{mm}$ negative artifact and a $6-\mathrm{mm}$ positive artifact. The mean pressure recorded by the digital equipment was an average of two artifacts. Similarly, the systolic pulmonary artery pressure contained a $24-\mathrm{mm}$ positive artifact, and the diastolic pressure contained a negative $22-\mathrm{mm}$ artifact. This mean pressure was also an average of two artifacts. Thus, for this patient, all of the information provided by the digital equipment was incorrect.

Figure 4 shows catheter whip. The pulmonary artery tracings demonstrate a spike-like deformity best seen in diastole, which is attributable to catheter whip. This spike covers very little actual area in the trace, but would be detected by the digital monitoring equipment and labeled as diastolic pressure. The spike deformity is presumed to be caused by motion of the catheter during the cardiac cycle, and is dampened when the catheter is relatively fixed in the wedge position. The sharply pointed systolic peak may represent catheter whip as well. The pulmonary artery wedge pressure tracing on the right shows less distortion.

Figure 5 shows combined catheter whip and inspiratory artifact. The patient had prosthetic mitral and aortic valves. In the systolic and diastolic tracings from the pulmonary artery, one can see the prominent positive and negative spikes that cover essentially no area on the tracing, but which caused marked negative and positive artifacts. On the wedge tracing one can see a 14$\mathrm{mm}$ deflection caused by the negative intrapleural pressure resulting from the patient's attempts to breathe.

Figure 6 shows automatic positive end-expiratory pressure (auto-PEEP). The patient had tachypnea, labored breathing and laryngeal edema. The pulmonary artery pressure tracing shows a typical positive and negative respiratory artifact $(\mathrm{A})$, as well as the very narrow negative spike that indicates catheter whip. Note that in this case, because the patient was breathing spontaneously, the positive artifact was not caused by a respirator but rather by the patient's attempts to exhale against an obstructed larynx (auto-PEEP). This situation produced a prolonged expiratory phase best seen in 


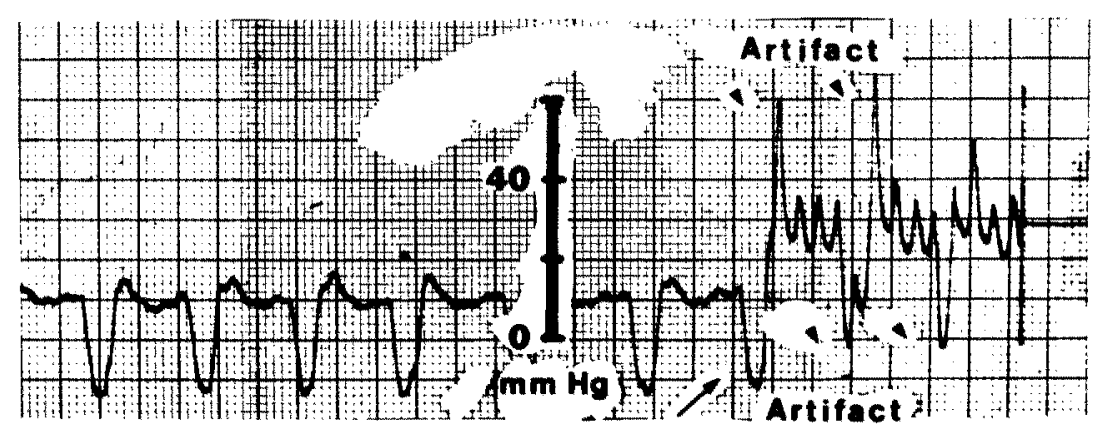

Fig 3. A recording from a patient with labored respiration, stiff lungs, and assisted ventilation, producing both positive and negative artifacts. The catheter balloon is inflated in the left tracing and deflated in the right tracing.

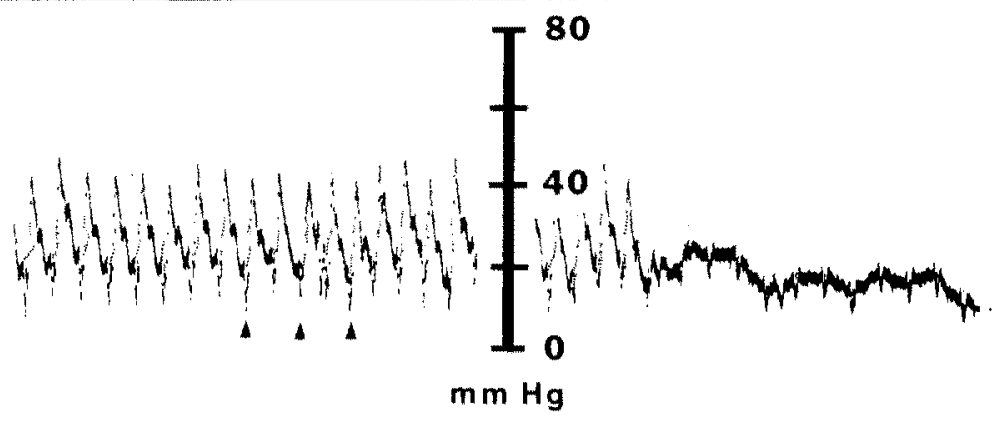

Fig 4. Negative artifact from catheter whip. The catheter balloon is inflated in the right tracing and deflated in the left.

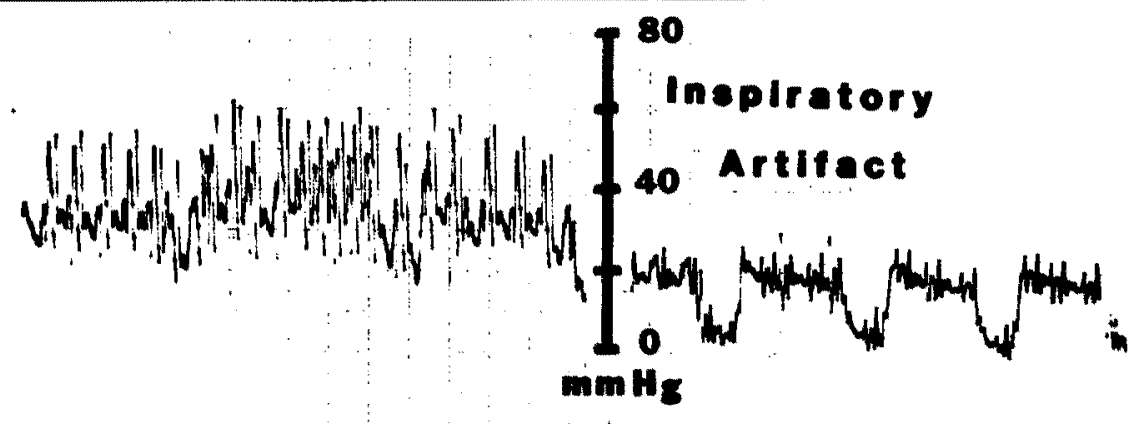

Fig 5. Recording showing catheter whip and inspiratory artifact. The balloon is deflated in the left tracing and inflated in the right.

the wedge pressure tracing. We would prefer to read the wedge pressure (PAWP) at the end of expiration, but the point where intrapleural pressure approximates atmospheric pressure occurs during such a brief time that it is difficult to find that exact spot. The arrow indicates where we think it is located in this tracing.

Figure 7 shows $V$ waves. This tracing was obtained from a patient in congestive heart failure. When the catheter balloon was properly inflated, the pressure peaks never entirely disappeard. This tracing was taken from a patient's chart, and shows how an inexperienced observer, who did not recognize the presence of abnormal $\mathrm{V}$ waves, tried to obliterate them by overinflating the balloon. This maneuver is dangerous to the patient, first because it may lead to pulmonary artery rupture and second because the data obtained are not related to the patient's hemodynamic state. Note that the pulmonary artery wedge pressure recorded by the observer $(40$ $\mathrm{mm} \mathrm{Hg}$ ) was higher than the pulmonary artery diastolic 


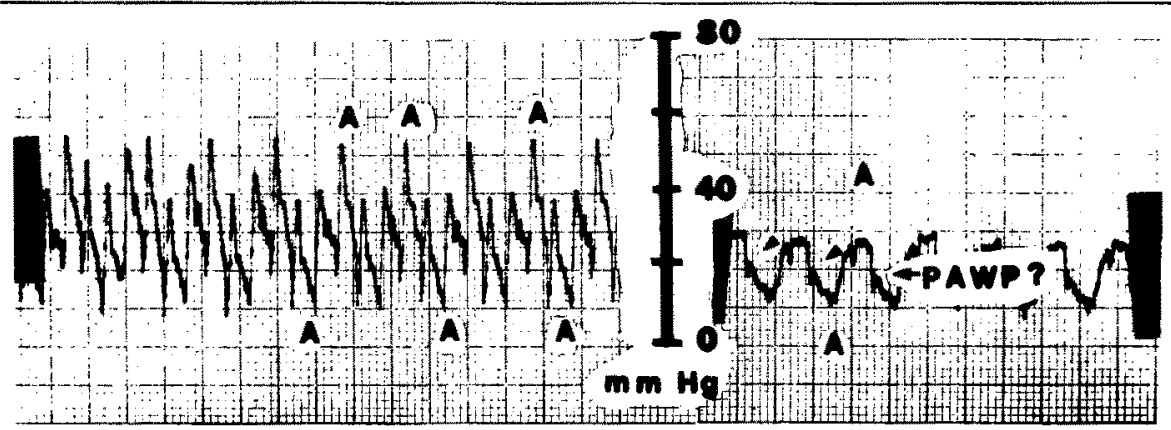

Fig 6. Positive and negative artifacts resulting from labored breathing, automatic positive end-expiratory pressure, tachypnea, and catheter whip. The balloon is deflated in the left tracing and inflated in the right. The arrowhead indicates our best estimate of true pulmonary artery wedge pressure (PAWP). A indicates positive and negative artifacts.
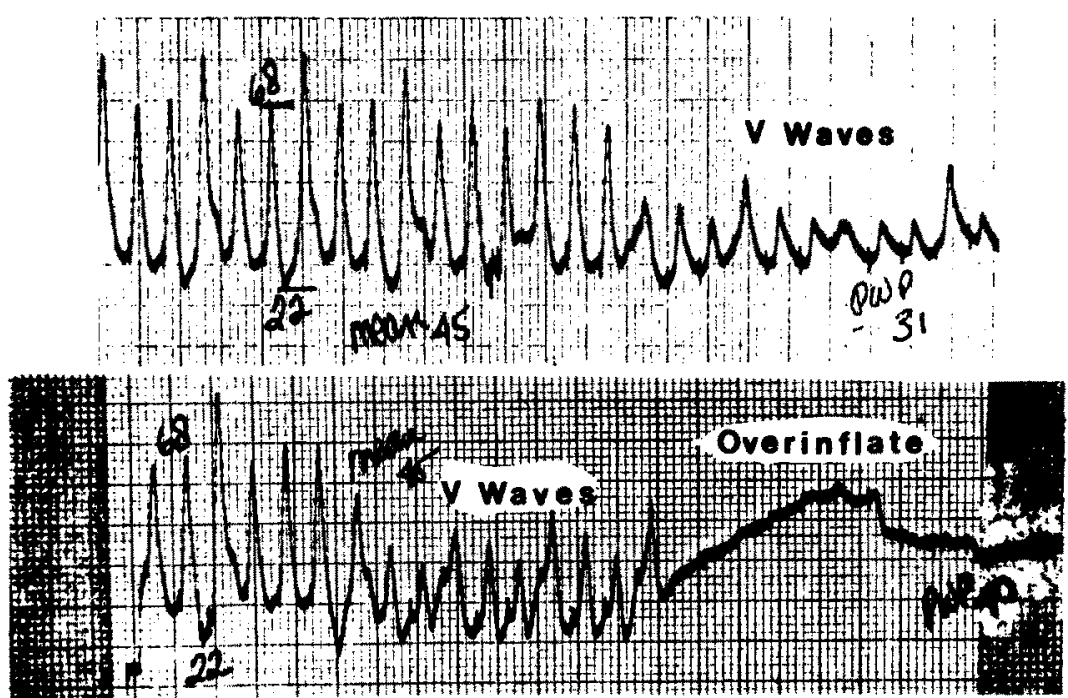

Fig 7. Tracings show $V$ waves and results of a dangerous attempt to compensate for them by overinflation of the catheter balloon. The balloon is deflated in the upper left half of the tracing and inflated in the upper right half. In the lower tracing the balloon is deflated in the left third of the tracing, inflated during the middle third, and overinflated in the right third.

pressure. In interpreting left ventricular filling pressures such as this one, the observer should ignore the $V$ wave and not try to obliterate it.

Figure 8 shows positive and negative respiratory artifacts associated with $V$ waves. In this example, the respiratory artifacts in the pulmonary artery wedge pressure tracing are the same as those seen in the previous examples. The tracing has a large $V$ wave that is seen in systole. When the balloon was inflated, the systolic pressure variation was not dampened out, and the digital electronics averaged the peak of the $V$ wave with the negative respiratory artifact to obtain an artifactually low value for the pulmonary artery wedge pressure.

Figure 9 shows inspiratory artifact and an overinflated catheter balloon. In this example the typical appearance of the rapidly rising pulmonary artery wedge pressure tracing seen in the right side of the diagram is a characteristic example of overinflation of the pulmonary artery balloon. The mechanism for this characteristic pattern is unclear; either the end of the catheter was occluded by being forced into the pulmonary artery wall or the balloon tip herniated over the end of the catheter. In either case, pressure in the flow device or in the balloon was measured, rather than pressure in the patient. On the left side of the tracing, however, there is a saw-toothed pattern that was produced by an inexperienced observer who knowingly attempted to overinflate the balloon 


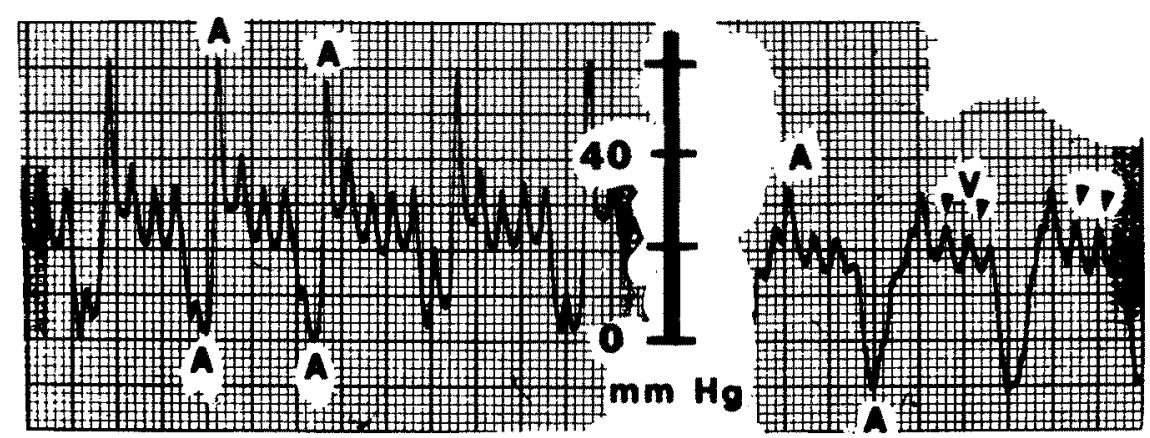

Fig 8. Positive and negative respiratory artifacts (A) assaciated with $V$ waves. The catheter balloon is deflated in the left side of the tracing and inflated in the right side.

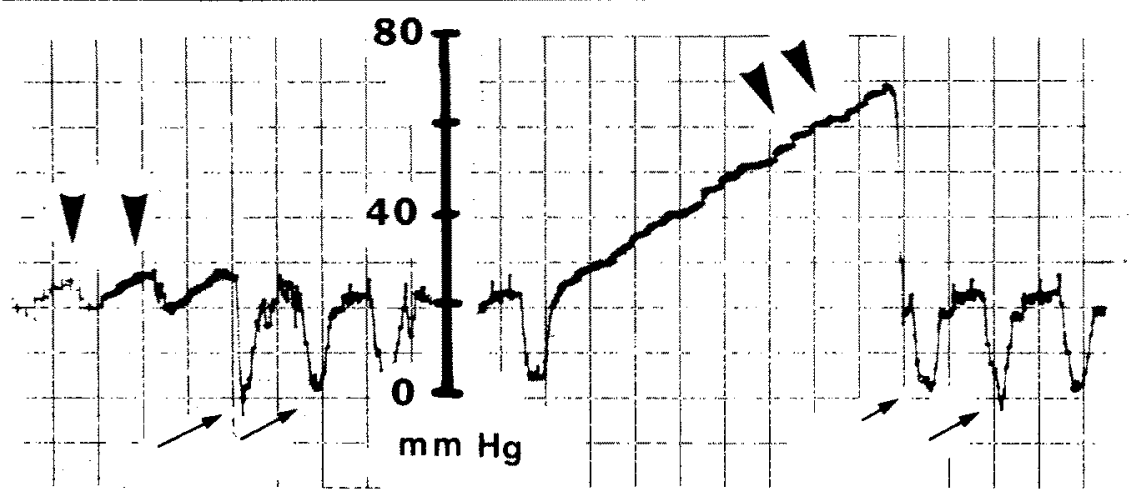

Fig 9. Inspiratory artifacts (small arrows) and attempts to compensate for them by overinflation of the balloon (large arrowheads). The balloon catheter is overinflated briefly in the left tracing, then deflated slightly to obtain a true pulmonary artery wedge pressure tracing that was not recognized; thus, the balloon was again overinflated. When the problem was recognized the balloon was deflated to an appropriate level.

enough to eliminate the unrecognized inspiratory artifact. At the extreme right side of the diagram is the true pulmonary artery wedge pressure tracing, with the prominently displayed typical negative respiratory artifact. We present this example so that this waveform may be instantly recognized and the balloon immediately deflated when this dangerous situation is recognized.

Figure 10 shows hiccoughs and the pulmonary artery wedge pressure. This example demonstrates the characteristic distortion of the pulmonary artery wedge pressure tracing produced by hiccoughs. A negative respiratory artifact can be seen as well. Although distortions produced in this way would seem self-explanatory, in this patient these artifacts produced erroneous digital data that was accepted as valid by the staff.
Figure 11 shows pulsus paradoxus. This example illustrates the moment-to-moment variation in the stroke volume of a patient in congestive heart failure. One must be arbitrary in selecting the point on the tracing that represents the pulmonary artery wedge pressure, and then be consistent in interpreting subsequent tracings. In this example, the point selected by the digital equipment is probably as consistent and accurate as that selected using analog data.

Figure 12: This example shows a chaotic respiratory pattern that occurred while the patient was receiving mechanical ventilation. Observation of the patient was required to correlate the various positive and negative pressure spikes with respiration. However, our final conclusion was that the pulmonary artery systolic and diastolic data were uninterpretable. The wedge pressure tracing, on the other hand, was interpretable. The positive spikes seen on the tracing are artifacts caused by the cycling of the respirator and the resulting positive intrapleural pressure. The negative spikes represent the patient's attempts at inspiration. When these attempts were not vigorous enough to initiate a respirator cycle, they were followed by a plateau rather than by a positive artifact. Thus the true pulmonary artery wedge 


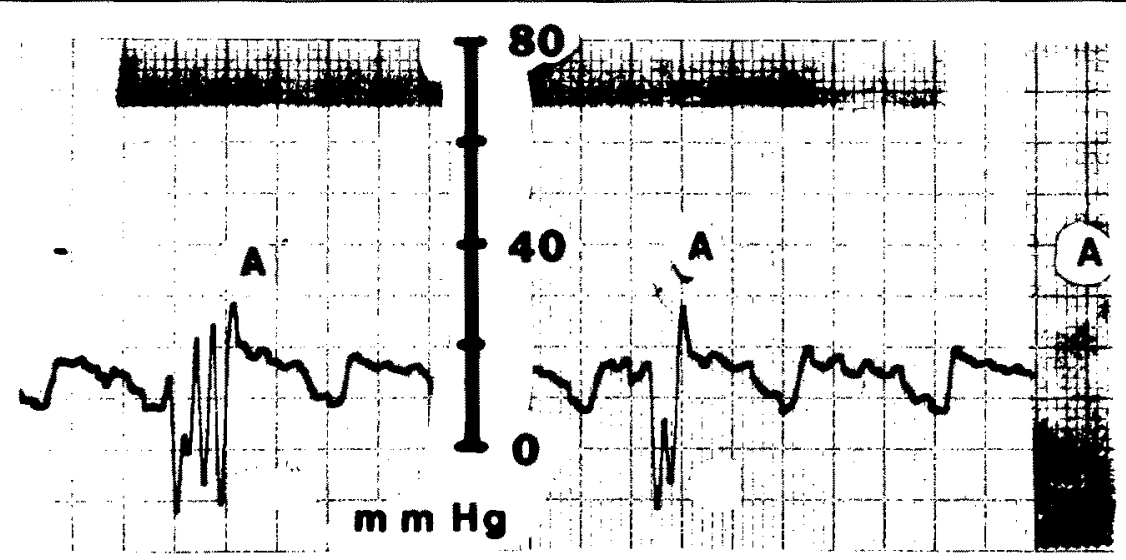

Fig 10. Tracing shows hiccoughs (A) and pulmonary artery wedge pressure. The catheter balloon is inflated throughout the tracing.

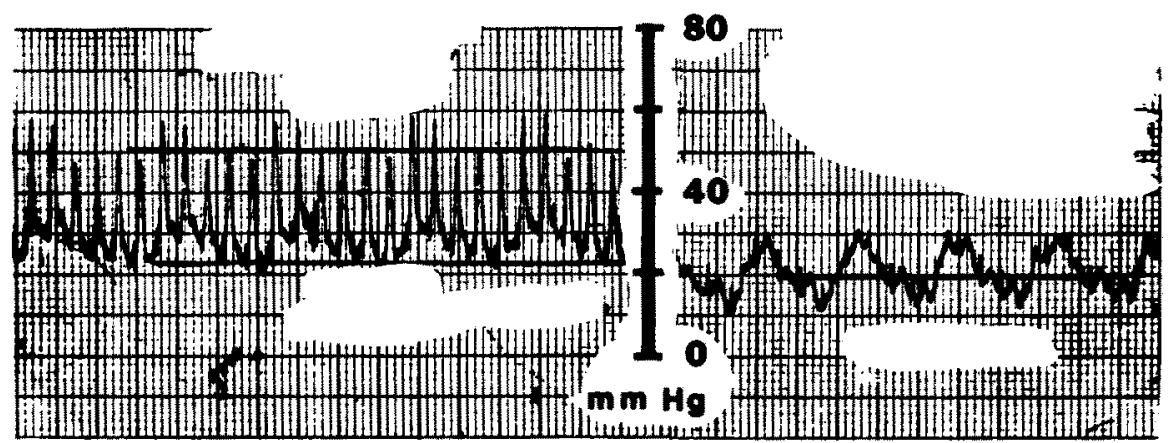

Fig 11. Tracing shows pulsus paradoxus. The lines indicate an approximation of pulmonary artery systolic, pulmonary artery diastolic, and pulmonary artery wedge pressures. The catheter balloon is deflated in the left side of the tracing and inflated in the right.

pressure in this patient was approximately $6 \mathrm{~mm} \mathrm{Hg}$, represented by the plateau seen in the tracing. Continuous display of airway pressure would have made interpretation easier, as the plateau could have been identified quickly as end expiration.

\section{DISCUSSION}

To understand the physiological characteristics of a digital readout is to understand how the machine arrives at the numbers that it displays. The monitors in use at Denver Presbyterian Hospital are typical of those used in many hospitals. They examine the output of the transducer for a discrete 4-second period of time known as the "scanning interval" and then calculate and display values obtained from this time period. They update and display the data for each new scanning interval. In this equipment, the scanning interval is almost, but not ex- actly, the time required for one screen width of analog pressure data. The machine then takes the lowest value observed and designates it as the "diastolic" value. It takes the highest value and designates it as the "systolic" value. The "mean" is an arithmetic average over time. If the lowest value seen is an artifact, then the value displayed for the "diastolic" value will be an artifact. If the highest value is an artifact, then the "systolic" value will be an artifact. If both are artifacts, then the mean will be an arithmetic average including two artifacts.

More sophisticated computer algorithms have been devised to deal with positive and negative artifacts, but they do not deal with many of the more complex combinations of artifacts shown here, nor do they call attention to changes in artifacts. Hence, even state-of-the-art algorithms are not reliable when compared with analog waveform interpretation by an experienced observer. However, they are probably more accurate than observations made by the usual monitor user.

The normal pulmonary artery wedge pressure tracing is not a straight line, but is made up of $\mathrm{A}, \mathrm{C}$, and $\mathrm{V}$ waves, which can be seen in most patients if the monitoring system has sufficient fidelity. For this reason, 


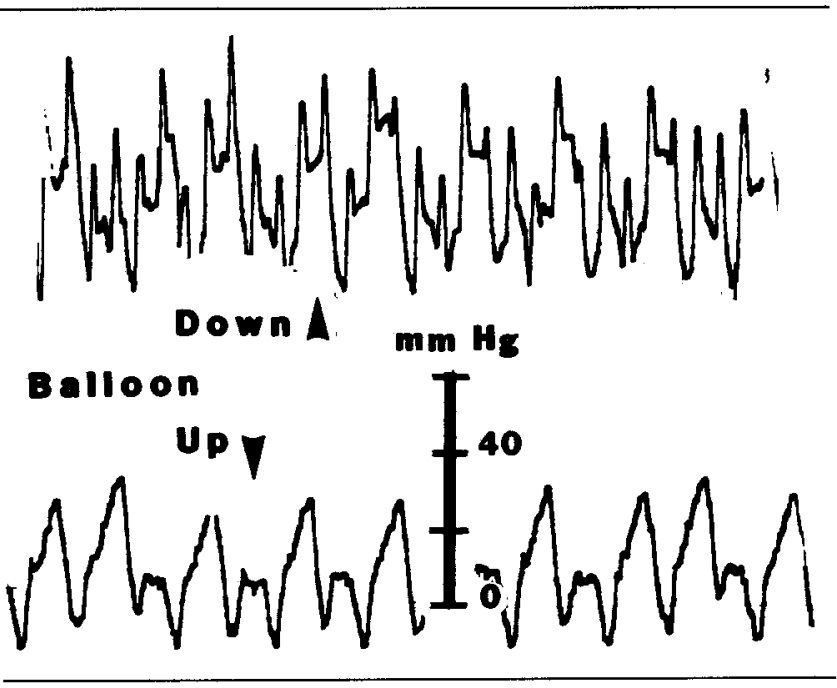

Fig 12. Tracings from a patient receiving mechanical ventilation. Airway pressure measurement would have helped in this example. The catheter balloon is deflated in the top tracing and inflated in the bottom tracing.

therapy is usually based on the mean value. The reference point for pressure values is atmospheric pressure. Any pressure that is present at the catheter tip, be it intrapleural or intravascular pressure, is measured by the transducer, compared to atmospheric pressure, and displayed on the screen. Large negative excursions in pleural pressure may be produced by a patient making a great effort to inspire. Large positive excursions may be produced by the respirator struggling against stiff lungs or by a patient exhaling against an obstructed larynx (auto-PEEP) [3]. (End expiration is considered the optimum time for measuring pulmonary artery pressures, because intrathoracic pressure is closest to atmospheric pressure at end expiration.) Other changes, such as pulsus paradoxus, that cause actual variations in cardiac output are more complicated [4] and require individual attention. When large $\mathrm{V}$ waves are superimposed on such a pattern the situation is even more complex.

Other authors have documented the effects reported here [5-12]. Some authors have described one or more artifacts and discussed their physiological characteristics. Others have provided normative statistical information about how incorrect the monitoring information may be. Our approach is more patient-specific. We have documented the important patterns of artifact and have provided a practical and inexpensive approach to handle them.

Berryhill and colleagues [7], in an elegant discussion, documented the effects of pleural pressure fluctuations on pulmonary artery pressure readings. They recommended the use of a chart recorder and measurements at end expiration. They also recommended continuous monitoring of airway pressure so that end expiration could be identified. In our experience, however, the end of expiration is usually easily identified without such monitoring.

Manipulations of the patient or the respirator have been proposed to eliminate intrapleural pressure artifacts. Davison and associates [8] measured pulmonary artery wedge pressure at end expiration in ventilated patients and compared this value with that obtained when patients were disconnected from the ventilator. The values were the same. Although their conclusion that pulmonary artery wedge pressure should be measured at end expiration, rather than when patients were disconnected from the respirator, was valid, their determination that pulmonary artery wedge pressure was the same when patients were and were not receiving mechanical ventilation simply indicated that their patients were not critically ill. Mechanical ventilation may produce important pulmonary artery and pulmonary artery wedge pressure changes that are not artifactual. Thus the physiological characteristics of patients receiving mechanical ventilation should be defined while they are being ventilated. Many patients cannot tolerate even a brief interruption of ventilatory support.

Schuster and Seeman [9] proposed that patients be paralyzed briefly to obtain accurate pressure data. This is another intervention that might change the patient's physiological status. Elimination of muscle tone can be expected to decrease venous return and make the pulmonary artery wedge pressure lower, as it did in their study. Physicians must take care that attempts to remove measurement artifacts do not in themselves produce misinformation.

Our purpose is to provide examples of most of the common and some of the uncommon patterns of pulmonary artery pressure seen in critically ill patients. It is important that physicians caring for these patients recognize from waveform patterns displayed on the monitor screen when these patterns will not be interpreted accurately by the digital display. Our preferred approach when such a pattern is recognized is to obtain a calibrated analog waveform, either by a strip-chart recorder or, if the monitors are capable of generating a calibrated graphic display of waveforms, by directly interpreting the waveform from the monitor. The calibrated strip-chart recorder is preferable because it allows more leisurely study of the waveforms and provides a permanent record for retrospective review and possible reconsideration. A chart recorder is inexpensive and can be interfaced with most monitoring equipment. In addition, it can be moved from patient to patient as needed. 


\section{REFERENCES}

1. Swan HJC, Ganz W, Forrester J, et al: Cardiac catheterization with a flow directed, balloon tipped catheter. N Engl J Med 1970;283:447-451

2. Ellis DM: Interpretation of beat-to-beat blood pressure values in the presence of ventilatory changes. J Clin Monit $1985 ; 1: 65-70$

3. Pepe PE, Marini JJ: Occult positive end-expiratory pressure in mechanically ventilated patients with airflow obstruction. Am Rev Respir Dis 1982;126:166-170

4. McGregor M: Pulsus paradoxus. N Engl J Med 1979; $301: 480-482$

5. Rice DL, Awe RJ, Gaaseh WH, et al: Wedge pressure measurement in obstructive pulmonary disease. Chest 1974;66:628-632

6. Permutt S: Relation between pulmonary arterial pressure and pleural pressure during the acute asthmatic attack. Chest 1973;63:25S-28S

7. Berryhill RE, Benumot JL, Rauscher LA: Pulmonary vascular pressure reading at the end of exhalation. Anesthesiology 1978;49:365-368

8. Davison D, Parker M, Harrison RA: The validity of determination of pulmonary wedge pressure during mechanical ventilation. Chest 1978;73:352-355

9. Schuster DP, Seeman MD: Temporary muscle paralysis for accurate measurement of pulmonary artery occlusion pressure. Chest 1983;84:593-597

10. Maran AG: Variables in pulmonary capillary wedge pressure: Variation with intrathoracic pressure, graphic and digital recorders. Crit Care Med 1980;8:102-105

11. Quinn KQ, Quebbeman EJ: Pulmonary artery pressure monitoring in the surgical intensive care unit. Arch Surg $1981 ; 116: 872-876$

12. Cengiz M, Crapo RO, Gardner RM: The effect of ventilation on the accuracy of pulmonary artery and wedge pressure measurements. Crit Care Med 1983;11:502-507 